Meta

Journal des traducteurs

Translators' Journal

\title{
La révision didactique
}

\section{Paul A. Horguelin}

Volume 33, numéro 2, juin 1988

L'enseignement de la traduction au Canada - Teaching Translation

in Canada

URI : https://id.erudit.org/iderudit/003112ar

DOI : https://doi.org/10.7202/003112ar

Aller au sommaire du numéro

Éditeur(s)

Les Presses de l'Université de Montréal

ISSN

0026-0452 (imprimé)

1492-1421 (numérique)

Découvrir la revue

Citer cet article

Horguelin, P. A. (1988). La révision didactique. Meta, 33(2), 253-257.

https://doi.org/10.7202/003112ar

Ce document est protégé par la loi sur le droit d'auteur. L'utilisation des services d'Érudit (y compris la reproduction) est assujettie à sa politique d'utilisation que vous pouvez consulter en ligne.

https://apropos.erudit.org/fr/usagers/politique-dutilisation/ 


\section{LA RÉVISION DIDACTIQUE}

PAUl A. Horguelin

Université de Montréal, Montréal, Québec

L'une des particularités de l'enseignement de la traduction au Canada est d'être, somme toute, proche des réalités professionnelles, tant par l'orientation des programmes que par la composition des corps enseignants. Il n'est donc pas surprenant que la création des premiers cours de révision ait suivi de près le "boum de la traduction ", au début des années 70 . Le recrutement intensif de traducteurs ayant engendré un besoin d'encadrement, quelques diplômés frais émoulus de l'université se retrouvèrent réviseurs sans avoir reçu une formation les préparant à cette fonction. Ainsi apparut la nécessité de donner aux étudiants en traduction des notions de révision de textes.

\section{HISTORIQUE}

C'est à l'automne de 1973 que l'assemblée des professeurs de l'École de traduction de l'Université de Montréal décide d'ajouter au programme de maîtrise, créé en 1971, un cours obligatoire intitulé "Technique de la révision". Dès le départ, le contenu de ce cours révèle la dimension professionnelle qu'il a gardée jusqu'à ce jour : "Théorie et pratique de la révision. Milieu de travail, conditions psychologiques et statut du réviseur. Exercices pratiques. " Parallèlement, la révision fait une entrée timide dans le programme de baccalauréat spécialisé, prenant place - à côté de la traduction au magnétophone - dans un séminaire à option. (Celui-ci sera remplacé en 1978 par un cours obligatoire : Initiation à la révision.) À la rentrée de 1974, les étudiants des deux cycles se voient donc offrir un cours de révision ; c'est une première au Canada et, sauf erreur, dans le monde.

Rapidement, l'initiative allait faire boule de neige. Dès 1975 , les étudiants anglophones de l'École de traduction reçoivent à leur tour la bonne parole dans un cours de Bilingual Revision. Puis, c'est la faculté de l'éducation permanente qui entre dans la ronde : création d'un cours d'Initiation à la révision en 1978, auquel viendra s'ajouter en 1980 - à la demande des étudiants inscrits au certificat en traduction - un cours de révision II. Progressivement, la plupart des écoles de traduction canadiennes inscriront la révision à leurs programmes, confirmant son accession au rang de discipline d'enseignement. Signalons enfin que la Société des traducteurs du Québec a offert à ses membres, en 1978, un cours de révision et, l'année suivante, organisé une journée d'étude sur la révision, en collaboration avec l'Association canadienne des écoles de traduction.

\section{OBJECTIFS}

Il est peut-être paradoxal, mais non moins évident, qu'un cours de révision ne vise pas à former des réviseurs - sauf exceptionnellement, s'il s'adresse à des traducteurs en exercice. Quelques heures d'enseignement ne peuvent suppléer à des années d'expérience. Les objectifs sont à la fois plus modestes et plus diversifiés.

Dans le cas de la révision bilingue, seule envisagée à l'origine, il s'agit bien entendu de familiariser l'étudiant avec une étape du processus de la traduction. Certains diplômés deviendront effectivement réviseurs, d'autres pratiqueront l'autorévision, presque tous seront révisés. Il importe donc de les préparer à cette réalité, qui a deux facettes : l'une est technique - comment procéder (mode de notation des corrections) et 
selon quels critères ; l'autre, également importante sur le plan professionnel, touche la délicate question des rapports entre réviseurs et révisés. Mais l'enseignement de la révision s'est aussi révélé un excellent moyen de compléter la formation des traducteurs. Confronté à une traduction qui n'est pas la sienne (ni le "corrigé » du professeur), et qui présente l'avantage d'être authentique, l'étudiant peut la décortiquer à son aise, enregistrant les passages bien rendus ou, au contraire, s'efforçant de remédier aux déficiences. Il est de ce fait amené à tester in vivo son apprentissage de la traduction, et à accrô̂tre ainsi sa compétence, le cours de révision se situant en terminale. Enfin, les exercices de révision offrent à l'étudiant de multiples occasions d'affiner ce que nous appelons son "jugement linguistique ", chaque intervention ponctuelle l'obligeant à prononcer (et à justifier) un verdict : c'est bon, acceptable, incorrect. L'aspect le plus formateur du cours réside d'ailleurs dans cette contrainte, puisque la traduction est essentiellement affaire de choix, et donc de jugement.

Au fil des ans, les objectifs du cours de révision ont été élargis pour tenir compte de deux facteurs nouveaux. D'une part, en raison de l'évolution de la situation linguistique au Québec, il a fallu faire une place à la révision unilingue. En effet, l'importance nouvelle donnée à la rédaction, notamment dans le cadre des programmes de francisation des entreprises, a ouvert l'éventail des possibilités de carrière offertes aux diplômés : rédaction, aide à la rédaction, conseil linguistique. Or, les cours de révision peuvent préparer directement à cette orientation, qu'on envisageait à peine au moment où ils furent créés. D'autre part, les retombées des recherches en théorie de l'information, survenues au moment où traducteurs et rédacteurs prenaient davantage conscience de leur rôle de communicateur, ont influé sur les paramètres de la révision, auxquels sont venus s'intégrer des critères de lisibilité et d'efficacité de la communication.

Il existe deux modes de révision, qui sont fonction des fins poursuivies. La révision pragmatique ne vise qu'à rendre un texte conforme à certains critères, sans qu'il y ait communication entre réviseur et révisé. S'il s'agit en plus d'assurer le perfectionnement de rédacteurs ou traducteurs en cours d'emploi, ou bien d'apporter un complément de formation à des étudiants, la révision est alors didactique. C'est évidemment cette dernière qui nous concerne ici.

\section{DESCRIPTION DU COURS}

Le cours de révision est essentiellement axé sur des exercices pratiques, la partie théorique se limitant à quelques exposés que viennent compléter des lectures recommandées. L'utilisation d'un manuel permet évidemment de consacrer plus de temps aux exercices, le professeur se contentant alors d'insister sur les points importants et de fournir les précisions demandées par les étudiants. Voici les principaux thèmes abordés. Viennent en premier lieu une définition des différents types de contrôle de la qualité des textes, un bref historique de la révision et une revue synthétique des méthodes d'évaluation. Cette introduction sert de toile de fond à une étude des principes généraux et de la technique de la révision, partie essentielle du cours : choix des paramètres, principes directeurs, pièges à éviter, règles et modes de notation, études de cas. La révision est ensuite envisagée dans sa dimension professionnelle : importance des relations humaines et conditions d'exercice de la profession. Enfin, le cours comprend une initiation à la correction d'épreuves : outre que la connaissance du code typographique doit faire partie de la formation des traducteurs et des rédacteurs, il importe de différencier les modes de notation utilisés en révision et en correction d'épreuves.

Les travaux pratiques se présentent sous trois formes. La majeure partie est, comme il se doit, constituée d'exercices de révision. Ceux-ci portent sur des textes authentiques, originaux ou traduits, appartenant à différents types de communication (lettres d'affaires, annonces, modes d'emploi et directives, rapports, articles de journaux et 
de revues...) et provenant de divers secteurs d'activité. Sauf exception, ce ne sont pas des modèles de prose, car la tâche de l'étudiant-réviseur se limiterait à manifester son appréciation... Pour éviter tout risque d'équivoque, et d'autosatisfaction, le professeur doit donc préciser que la qualité des textes à réviser n'est pas de niveau professionnel (même si certains sont, de fait, l'œuvre de "professionnels "). Un autre type d'exercice consiste à corriger des épreuves de textes imprimés : les étudiants apprennent ainsi à utiliser le code typographique, tout en découvrant que la correction d'épreuves est un art plus difficile qu'ils ne le pensaient. Enfin, une série d'exercices porte sur les fautes les plus fréquentes relevées dans des écrits publiés au Canada (orthographe, accord, structure, niveaux, anglicismes, barbarismes...). S'y ajoutent quelques énoncés contenant des points litigieux, principalement des écarts entre la norme et l'usage, pour lesquels l'étudiant doit exercer son jugement. Au total, ces exercices de correction permettent de passer en revue quelque trois cents fautes courantes.

\section{DÉROULEMENT D'UN COURS}

Entrons dans la classe pour "assister " à un cours type (durée : 2 h 40). On commence par un exercice de correction, en guise de mise en train. Comme il s'agit d'un simple contrôle des connaissances (la plupart des difficultés ont normalement été signalées dans d'autres cours), l'exercice se fait en temps limité. La correction orale suit immédiaternent, accompagnée des observations et justifications appropriées. Vient ensuite la période de commentaires et de questions sur le chapitre du cours que les étudiants avaient à lire. Elle dépasse rarement le quart d'heure, ce qui permet de passer sans retard au "plat de résistance" : les exercices de révision.

Pour chaque classe, il est proposé aux étudiants un travail pratique (TP) qui consiste à réviser un texte de deux pages à double interligne. Ici s'imposent quelques précisions sur le système de notation que nous employons. Étant donné qu'il s'agit de révision didactique, il nous a paru utile d'établir une distinction entre les corrections, notées au stylo rouge (tradition oblige!) et les améliorations, indiquées en vert. Est considéré comme justifiant une correction tout écart à la norme, attesté par les dictionnaires ou grammaires (au besoin, on peut demander aux étudiants de citer leurs sources). Les faux sens et contresens entrent aussi dans cette catégorie. Quant aux améliorations, elles visent principalement à accroître la lisibilité et la qualité stylistique du texte, sans que les termes ou tournures en cause soient incorrects. Cette distinction n'est pas un pur raffinement. Elle habitue les étudiants à ne pas confondre erreur grave et peccadille, alors qu'on constate dans leurs premiers travaux qu'ils voient presque toujours la paille, mais plus rarement la poutre. De plus, elle les prépare à des situations où la contrainte du temps ne leur laissera pas le loisir de fignoler. Enfin, elle les incite à respecter l'autonomie professionnelle du révisé qui, s'il ne peut contester les corrections, devrait avoir la latitude de juger la pertinence des améliorations suggérées.

Comment s'effectue la correction des TP ? Deux formules sont envisageables. Dans le premier cas, le professeur corrige lui-même les travaux et les remet à la classe suivante. Inconvénients : le temps de correction est extrêmement long, puisqu'il faut travailler sur deux textes - l'original et la version révisée (et même trois, s'il s'agit d'une révision bilingue) ; en outre, il y a un décalage d'au moins une semaine entre la remise du TP et sa correction en classe. Pour ces raisons, nous avons adopté le système de la correction réciproque : les étudiants échangent leurs travaux et jouent le rôle de correcteur. À l'usage, nous avons constaté que cette formule accroît la valeur formatrice de l'exercice, du fait que chaque étudiant, après avoir révisé le texte, en révise la révision faite par un autre. Quant au risque de "correction de complaisance ", disons qu'il est inexistant : deux contrôles effectués pendant les séances d'examen confirment chaque année que les étudiants pèchent plus par sévérité que par indulgence. 
Ces précisions apportées, retournons en classe pour la suite des opérations. Le professeur lit chaque phrase ou segment de phrase en indiquant séparément les corrections et les améliorations. Si la révision a été faite correctement, les étudiants inscrivent le pointage dans la marge ; dans le cas contraire, ils notent l'omission en interligne. En règle générale, les corrections ne prêtent pas à contestation, puisque par définition elle doivent pouvoir être justifiées - ce que fait le professeur, sources à l'appui. Il n'en va pas toujours de même dans le cas des améliorations... Certaines "passent " bien : choix d'un terme plus précis, tournure plus concise, restructuration améliorant la lisibilité. Mais il arrive que les améliorations stylistiques fassent problème, car on conviendra qu'il est rarement possible de prouver, hors de tout subjectivisme, qu'une tournure est plus idiomatique qu'une autre, également correcte. Le professeur fait alors valoir ses arguments et tente d'établir un consensus ; en dernier recours, il doit trancher pour couper court aux palabres. C'est le seul cas, d'ailleurs assez rare, qui nécessite un acte d'autorité.

Â la fin de l'exercice, les étudiants totalisent les points (un point par correction et un demi-point par amélioration). Le professeur indique le coefficient permettant d'obtenir une notation sur 100 et ajoute une "bonification", en moyenne de 10 points, qui tient compte de l'écart de compétence entre l'apprenti réviseur et le réviseur professionnel. Chaque correcteur signe en regard du décompte, ce qui permet un contrôle ultérieur.

Les examens sont corrigés par le professeur, selon le même système. Toutefois, ce sont des épreuves en temps limité, pour rappeler aux étudiants qu'ils devront, dans la pratique professionnelle, tenir compte du facteur temps. En outre sont sanctionnées par des points en moins les fautes fictives (corrections non justifiées) et celles que l'étudiant introduit dans sa révision : dans ce cas, c'est la crédibilité du futur réviseur qui est en cause.

\section{BILAN}

En conclusion, l'expérience de plus de dix années d'enseignement de la révision nous autorise à dire que le bilan est très positif, tant sur le plan de la formation que sur celui de la motivation.

La variété des exercices proposés permet de passer en revue les principales difficultés de la langue et de la traduction à l'aide de cas concrets et réels. Les étudiants reconnaissent d'ailleurs, en fin d'année, que le cours offre une forte densité d'enseignements pratiques. Ils constatent aussi - comme leur professeur - que c'est un cours très exigeant, du fait de la nécessité d'être en mesure de justifier presque chaque intervention ponctuelle. De plus, étant donné que la consultation de nombreux ouvrages fait ressortir des divergences et même des contradictions, interviennent les questions de normes et d'usages - qui sont au cour même du travail de révision. D'où de fréquentes remises en cause (parfois déchirantes !) des idiolectes et, en fin de course, un affinement du jugement linguistique. Chemin faisant, l'étudiant prend aussi conscience de la dimension " relations humaines " de la révision, en vivant en classe des situations qu'il retrouvera plus tard dans son milieu de travail. Enfin, il reçoit une formation qui le prépare, directement ou indirectement, à plusieurs professions ou fonctions : révision, traduction, rédaction, aide à la rédaction, correction d'épreuves.

Le cours de révision suscite d'autre part une réelle motivation. Le fait qu'il soit "déculpabilisant " n'y est sans doute pas étranger. Les copies sont toujours raturées de rouge, mais ce sont cette fois les étudiants qui utilisent la couleur infamante et, qui plus est, sur des textes dont ils ne sont pas les auteurs. La vraie motivation, toutefois, réside ailleurs. Grâce à la correction réciproque - et en pratique, collective -, une classe de révision est un forum linguistique, presque un happening de la communication sur le 
vif. Confrontés au texte à réviser, puis à la révision qu'ils corrigent, enfin aux diverses opinions émises en classe, les étudiants ont la possibilité de s'exprimer, de défendre leur point de vue, de contribuer à l'amélioration de la version qui sera finalement retenue. Une fois établies les règles du jeu, chacun participe à un cours vivant, où l'on s'ennuie rarement.

\section{BIBLIOGRAPHIE SOMMAIRE}

DARBELNET, Jean (1978) : "Spécialisation et enseignement de la traduction", in la Traduction, une profession, Ottawa, CTIC, pp. 195-196.

HORGUELIN, Paul A. (1985): Pratique de la révision, $2^{e}$ éd., Montréal, Linguatech.

HIOSINGTON, B. and P. HORGUELIN (1980) : A Practical Guide to Bilingual Revision, Montréal, Linguatech.

JOYAL, Bernard (1969) : “Initiation à la traduction par la révision », META, 14:2, pp. 98-100.

THAON, Brenda (1984) : "The Role of a Revision Course in a Translation Program ", in la Traduction: l'universitaire et le praticien, Ottawa, Éditions de l'Université d'Ottawa, pp. 297-301. 